Artículo científico

Volumen 31(1): 193-208. Enero-abril, 2020

e-ISSN 2215-3608, doi:10.15517/am.v31i1.36677

http://www.revistas.ucr.ac.cr/index.php/agromeso

\title{
Producción y calidad de Tithonia diversifolia y Sambucus nigra en trópico altoandino colombiano ${ }^{1}$
}

\section{Production and quality of Tithonia diversifolia and Sambucus nigra high andean colombian tropic}

\author{
Carolina Guatusmal-Gelpud ${ }^{2}$,Laura Dayana Escobar-Pachajoa ${ }^{3}$, Diego Hernán Meneses-Buitrago², Juan \\ Leonardo Cardona-Iglesias ${ }^{2}$,Edwin Castro-Rincón ${ }^{2}$
}

1 Recepción: 27 de marzo, 2019. Aceptación: 9 de septiembre, 2019. Este trabajo formó parte del proyecto "Mejoramiento de la oferta forrajera, optimización de sistemas de alimentación y aseguramiento de la calidad e inocuidad de la leche en el trópico alto del departamento de Nariño", en la Corporación Colombiana de Investigación Agropecuaria (AGROSAVIA). Colombia.

2 Corporación Colombiana de Investigación Agropecuaria (AGROSAVIA). Nariño, Colombia. carolinagg080@gmail.com, lauraescp@gmail. com,dmeneses@agrosavia.co (https://orcid.org/0000-0003-3033-3079),jlcardona@agrosavia.co, ecastro@agrosavia.co (https:// orcid. org/0000-0001-9841-8242).

\section{Resumen}

Introducción. Entre las limitantes de los sistemas ganaderos del trópico alto colombiano está la estacionalidad forrajera. El uso de sistemas silvopastoriles podría maximizar la producción del alimento y garantizar oferta de nutrientes durante todo el año. Objetivo. Evaluar en Tithonia diversifolia y Sambucus nigra establecidas en un sistema silvopastoril, el efecto de dos edades y dos alturas de corte sobre variables morfométricas, producción y calidad nutricional. Materiales y métodos. El estudio se realizó en el Centro de Investigación Obonuco de la Corporación Colombiana de Investigación Agropecuaria (AGROSAVIA), Pasto, Colombia, entre enero y noviembre de 2018. Se estableció un diseño de bloques al azar en arreglo factorial con cuatro tratamientos (dos edades y dos alturas de corte) por cada especie (T1:60 días*20 cm, T2:60 días*50 cm, T3:80 días*20 cm, T4:80 días*50 cm para T. diversifolia y T1:60 días*30 cm, T2:60 días*50 cm, T3:90 días*30 cm, T4:90 días*50 cm para $S$. nigra). Resultados. Las variables productivas mostraron significancia en $T$. diversifolia con mayor materia seca (MS) a 80 días $\left(30,6 \mathrm{t} \mathrm{ha}^{-1}\right.$ año ${ }^{-1}$ ) y $S$. nigra a 90 días $\left(14,4 \mathrm{t} \mathrm{ha}^{-1} \mathrm{año}^{-1}\right)$; el número de ramas y rebrotes presentó mayores valores en $\mathrm{T} 4$ para $T$. diversifolia (127,10 ramas/arbusto y 819,65 rebrotes/arbusto). Las calidad nutricional de T. diversifolia y $S$. nigra fue significativamente mayor a los 60 días; con proteína cruda de 26,73\% y 26,25\%, digestibilidad de 75,45\% y 76,28 $\%$, y energía neta de lactancia de 1,58 y 1,60 Mcal kg-1 MS, respectivamente. Conclusión. La producción de biomasa (MS kg/arbusto) y capacidad de rebrote aumentaron cuando se cosechó a 80 días en T. diversifolia y 90 días en $S$. nigra; sin embargo, se presentó una mejor calidad nutricional a edades de corte de 60 días en el trópico alto de Nariño, lo que evidenció su potencial productivo para alimentación del ganado.

Palabras clave: sistemas silvopastoriles, forrajes, alimentación, edad, altura. 


\begin{abstract}
Introduction. Among the limitations of the livestock systems of the Colombian high tropic is fodder seasonality. The use of silvopastoral systems could be a strategy to maximize food production and guarantee a supply of nutrients throughout the year. Objective. To evaluate in Tithonia diversifolia and Sambucus nigra established in a silvopastoral system, the effect of two ages and cutting heights on morphometric, production, and nutritional quality variables. Materials and methods. The study was conducted at the Obonuco Research Center of the Corporacion Colombiana de Investigacion Agropecuaria (AGROSAVIA) (Pasto, Colombia) between January and November 2018. A random block design was established in factorial arrangement with four treatments (two ages and two cutting heights) for each species (T1:60 days $* 20 \mathrm{~cm}, \mathrm{~T} 2: 60$ days $* 50 \mathrm{~cm}, \mathrm{T3}: 80$ days $* 20 \mathrm{~cm}, \mathrm{~T} 4: 80$ days $* 50 \mathrm{~cm}$ for $T$. diversifolia and T1:60 days*30 $\mathrm{cm}, \mathrm{T} 2: 60$ days $* 50 \mathrm{~cm}, \mathrm{~T} 3: 90$ days $* 30 \mathrm{~cm}, \mathrm{~T} 4: 90$ days $* 50 \mathrm{~cm}$ for $S$. nigra). Results. The productive variables showed significance in $T$. diversifolia with higher dry matter (DM kg/bush) at 80 days (30.6 ton ha ${ }^{-1}$ year $\left.^{-1}\right)$ and $S$. nigra at 90 days (14.4 ton ha $^{-1}$ year $\left.^{-1}\right)$; the number of branches and shoots showed higher values in T4 for $T$. diversifolia (127.10 branches/bush and 819.65 shoots/bush). The nutritional quality of T. diversifolia and S. nigra was significantly higher at 60 days; with $26.73 \%$ and $26.25 \%$ crude protein, digestibility of $75.45 \%$, and $76.28 \%$, and net lactation energy of 1.58 and $1.60 \mathrm{Mcal} \mathrm{kg}^{-1} \mathrm{DM}$, respectively. Conclusion. Biomass production (DM) and resurgence capacity increased when harvested at 80 days in T. diversifolia and 90 days in S. nigra; however, a better nutritional quality was presented at cutting ages of 60 days in the high tropic of Nariño, which evidenced its productive potential for cattle feeding.
\end{abstract}

Keywords: silvopastoral systems, forages, food, age, height.

\title{
Introducción
}

En Colombia, los sistemas de producción de leche se establecen básicamente en el trópico de altura, que va desde los 1800 hasta $3000 \mathrm{msnm}$ (Morales et al., 2013); este tipo de sistemas ganaderos en general se caracterizan por una baja eficiencia en el uso del suelo, donde predomina el monocultivo de gramíneas, alta fertilización química y escasa implementación y uso de otras especies forrajeras para la alimentación del ganado (Santacoloma, 2011). Uno de los principales problemas de la ganadería en el trópico es la estacionalidad climática, debido a la fluctuación de los cambios ambientales, que puede provocar periodos marcados de épocas de lluvias o intensos veranos, situaciones que afectan directamente la oferta de forraje y de nutrientes (Matta, 2005; Roncallo et al., 2012). En estos sistemas de producción de leche se hace necesario hacer una reconversión integral, sobre todo referente al manejo y uso de recursos alimenticios, para así alcanzar impactos positivos a nivel ambiental y socioeconómico (Mahecha et al., 2002; Verdecia et al., 2011; Gallego-Castro et al., 2014).

La implementación de sistemas silvopastoriles (SSP) como cercas vivas, árboles dispersos en potrero o bancos forrajeros, presentan múltiples ventajas frente a sistemas tradicionales (Mohan y Ramachandran, 2011). En los bancos forrajeros (BF) se utilizan especies leñosas y herbáceas adaptadas a la zona, para corte y acarreo en altas densidades, tratando de garantizar el aporte de proteína, energía y fibra para los rumiantes (Murgueitio et al., 2011).

En el trópico altoandino del departamento de Nariño se evidencian dos aspectos que inciden sobre la promoción y uso eficiente de SSP; en primer lugar, no se encuentran muchas opciones de especies forrajeras para diversificar la oferta alimenticia de los animales. El otro factor se relaciona con el desconocimiento técnico de cuáles son las edades y alturas de corte óptimas al momento de la cosecha de las forrajeras. Factores como la edad de cosecha y altura de poda en especies forrajeras, determinan en gran medida la composición nutricional, producción de biomasa y velocidad de rebrote de la especie (Londoño et al., 2019). Lo anterior se corroboró en diversos estudios que mostraron un efecto marcado de condiciones de manejo como la edad de cosecha, altura de corte y distancias 
de siembra, sobre la respuesta productiva de especies como la Tithonia diversifolia (Jama et al., 2000; Gallego et al., 2015).

Especies como el botón de oro (Tithonia diversifolia) y el sauco (Sambucus nigra), podrían llegar a ser una opción en suplementación para rumiantes, sobre todo en épocas de escases de pasturas. La $T$. diversifolia es una especie de la familia Asteraceae, presenta alta producción de biomasa, con reportes de $19 \mathrm{t} \mathrm{ha}^{-1} \mathrm{MS}^{-1}$ al año (Gallego et al., 2015), es reconocida por su buen aporte proteico [(hasta $25 \%$ proteína cruda PC)] y contenido de aminoácidos esenciales en relación con la proteína total (Medina et al., 2009; Rivera et al., 2015). Además, presenta un buen contenido de extracto libre de nitrógeno (ELN), como el reportado por Cardona et al. (2017), de 29,5\% a una edad de 70 días de rebrote. T. diversifolia presenta valores de fibra en detergente neutro (FDN) entre 25,2 y $39 \%$ (Rivera et al., 2015; Cardona-Iglesias et al., 2017), rangos que sugiere el National Research Council (NRC, 2001) de una FDN apropiada (mínimo de $25 \%$ en la dieta) para estimular rumia y mantener activas las funciones ruminales (Banakar et al., 2018).

Otra especie con potencial productivo en el trópico alto colombiano es el S. nigra de la familia Adoxaceae; en el departamento de Nariño esta especie mostró contenidos de nutrientes importantes, tales como: $19 \%$ de materia seca (MS), 23,8 \% de proteína cruda (PC), 23,4\% de fibra en detergente neutro (FDN), 17,28\% fibra en detergente ácido (FDA), 11,2 \% de cenizas y 1,4\% de calcio (Ca) en relación con la MS (Apráez et al., 2012). También se han reportado valores de digestibilidad de la MS del 58 \% (Cárdenas et al., 2011). Para Cárdenas et al. (2016), las edades de corte del sauco, así como de otras especies forrajeras, determinan en gran medida las respuestas en producción de biomasa y de calidad nutricional.

La información resultante de evaluaciones en especies forrajeras adaptadas a la zona, sobre edades y alturas de cosecha óptimas, será de gran importancia para los productores locales. Además la adopción de SSP como bancos forrajeros, podría ser una alternativa viable para mejorar la oferta alimenticia de los animales, sobre todo en época de escases de pasturas.

El objetivo de este trabajo fue evaluar en Tithonia diversifolia y Sambucus nigra establecidas en un sistema silvopastoril, el efecto de dos edades y alturas de corte sobre variables morfométricas, de producción y calidad nutricional.

\section{Materiales y métodos}

\section{Localización}

El estudio se llevó a cabo en el Centro de Investigación (C.I) Obonuco perteneciente a la Corporación Colombiana de Investigación Agropecuaria (AGROSAVIA), ubicado en el municipio de Pasto, Colombia. El área de estudio estaba ubicada a $1^{\circ} 11^{\prime} 41,3^{\prime}$ N y $77^{\circ} 19^{\prime} 19^{\prime \prime}$, con una altitud de $2905 \mathrm{msnm}$, una precipitación promedio anual de $619,7 \mathrm{~mm}$, una humedad relativa de $84,4 \%$ y temperatura promedio de $12,8{ }^{\circ} \mathrm{C}$, valores que fueron registrados por medio de una estación meteorológica (Escobar-Pachajoa et al., 2019), durante el período de investigación correspondiente a once meses, entre enero y noviembre de 2018.

\section{Diseño experimental}

Se empleó un diseño de bloques completos al azar con un arreglo factorial $2 * 2$ con tres repeticiones y cuatro tratamientos por especie, derivados de la interacción entre los factores edad de corte y altura de corte (Cuadro 1). 
Cuadro 1. Tratamientos para cada especie arbustiva forrajera en banco forrajero establecido en el Centro de Investigación Obonuco perteneciente a la Corporación Colombiana de Investigación Agropecuaria (AGROSAVIA), Pasto, Colombia. 2019.

Table 1. Treatments for each forage bush species in fodder bank established in the Obonuco Research Center belonging to the Corporacion Colombiana de Investigación Agropecuaria (AGROSAVIA), Pasto, Colombia. 2019.

\begin{tabular}{cc}
\hline Tithonia diversifolia & \multicolumn{1}{c}{ Sambucus nigra } \\
\hline T1: 60 días de corte y $10 \mathrm{~cm}$ de altura & T1: 60 días de corte y $30 \mathrm{~cm}$ de altura \\
T2: 60 días de corte y $50 \mathrm{~cm}$ de altura & T2: 60 días de corte y $60 \mathrm{~cm}$ de altura \\
T3: 80 días de corte y $10 \mathrm{~cm}$ de altura & T3: 90 días de corte y $30 \mathrm{~cm}$ de altura \\
T4: 80 días de corte y $50 \mathrm{~cm}$ de altura & T4: 90 días de corte y $60 \mathrm{~cm}$ de altura \\
\hline
\end{tabular}

\section{Descripción del experimento}

La evaluación se realizó en un sistema silvopastoril (banco forrajero) compuesto por las especies botón de oro ( $T$. diversifolia) y sauco ( . nigra) las cuales tenían aproximadamente año y medio de establecidas, en un área total de $153 \mathrm{~m}^{2}$. Se encontraban sembradas en bloques con distancias de siembra de $1 \mathrm{~m}^{*} 1 \mathrm{~m}$, para un total de 102 arbustos (51 de T. diversifolia y 51 de S. nigra). Cada unidad experimental tenía un área de $9 \mathrm{~m}^{2}$ con 9 árboles evaluados por tratamiento. Se mitigó el efecto de borde con la separación de un surco de arbustos que no fueron evaluados. El periodo de evaluación se desarrolló desde enero hasta noviembre de 2018 con el corte de uniformización, y comprendió tres ciclos de producción, el primero entre marzo-mayo, el segundo entre junioagosto y el tercero entre septiembre-noviembre de 2018.

El manejo agronómico del experimento consistió en plateo de los arbustos cada dos meses a un radio de 40 $\mathrm{cm}$ del fuste, mediante la poda del pasto circundante, con guadaña y/o de forma manual. Complementariamente se realizó una aplicación preventiva con insecticida orgánico y se efectuó una fertilización orgánica y química de acuerdo con el análisis de suelos después de cada corte de uniformización.

\section{Variables evaluadas}

\section{Producción de biomasa (rendimiento)}

Se estimó la producción de forraje verde por medio de una poda manual de los arbustos marcados en cada parcela, para lo cual se realizó un corte de homogenización a una altura de $50 \mathrm{~cm}$ sobre el nivel del suelo (López y Santamaría, 2003). Posteriormente, se realizaron tres cortes (ciclos) para cada uno de los tratamientos por especie. Para las muestras se cosechó el total de hojas y tallos tiernos, a partir de tres arbustos que conformaron la unidad experimental por tratamiento. La biomasa resultante se pesó en una balanza de precisión VIBRA y se tabularon los datos en el programa Microsoft Excel ${ }^{\circledR}$. La materia seca (MS) se calculó en kg por arbusto, para ello se secó una muestra compuesta de $500 \mathrm{~g}$ de forraje verde hasta peso constante (Téllez y Mendoza, 2014); adicionalmente, se calculó el rendimiento de MS en $\mathrm{t} \mathrm{ha}^{-1} \mathrm{año}^{-1}$ con base en la edad, para determinar el número de cortes por año.

\section{Variables morfométricas}

Después de cada corte se realizaron tres mediciones cada 15 días de: diámetro de copa (con cinta métrica, de acuerdo con lo señalado por Corvalán y Hernández (2012), número de ramas (conteo visual), número de rebrotes 
(conteo visual), número de botones (conteo visual) y vigor, este último expresado por el estado de la planta, color, crecimiento y sanidad en una escala de 1 a 5, donde 1 susceptibles, 2 tolerantes, 3 resistentes, 4-5 plantas sanas o inmunes, teniendo en cuenta como parámetro de comparación el cultivo en general (Medina et al., 2009).

\section{Calidad nutricional}

Se realizaron análisis de composición nutricional para ambas especies arbustivas, a través de la metodología de espectrofotometría por infrarrojo cercano (NIRS), con la cual se determinó porcentaje (\%) de proteína cruda (PC), fibra en detergente neutro (FDN), fibra en detergente ácido (FDA), lignina, hemicelulosa, calcio (Ca), fósforo $(\mathrm{P})$, nutrientes digestibles totales (NDT), digestibilidad y energía expresada como: $\mathrm{Mcal} \mathrm{kg}^{-1} \mathrm{MS}$ de energía neta de lactancia (ENL).

\section{Análisis estadístico}

Se dividió el análisis en variables cuantitativas (diámetro de copa, número de rebrotes y número de botones) y variables cualitativas (vigor). Posteriormente, los datos se analizaron con base en su varianza y distribución normal, y el empleo del paquete CAR (Fox y Weisberg, 2011), para determinar diferencias significativas entre los tratamientos. Los datos de producción por arbusto, rendimiento $\mathrm{t}^{-1} \mathrm{anno}^{-1} \mathrm{y}$ de composición se analizaron por medio de análisis de varianza, acompañados de la prueba de comparación de medias de Tukey $(\alpha=0,05)$; en caso contrario, para determinar el efecto de los factores por separado y teniendo en cuenta que los niveles de los factores son cualitativos, se realizó un análisis de contrastes octogonales $(\alpha=0,05)$. La variable vigor al ser categórica- politómica-ordinal se analizó a través de tablas de contingencia acompañadas del estadístico de Chi-Cuadrado de Pearson $(\alpha=0,05)$, buscando determinar la relación de dependencia con los tratamientos. Los cálculos se realizaron con base en el software estadístico R. V.3.5.1 (R Development Core Team, 2008), utilizando el paquete agricolae (Mendiburu, 2017).

\section{Resultados}

Durante el año de evaluación se presentaron tres épocas definidas, dos de altas precipitaciones y otra de bajas precipitaciones, la temperatura media varió entre los 11 y $12{ }^{\circ} \mathrm{C}$. La distribución de estas variables a través del tiempo para la zona de Pasto se puede observar en la Figura 1.

\section{Producción, rendimiento y variables morfométricas}

En las variables de producción FV y MS no se presentó significancia en el tratamiento, mientras que en las variables morfométricas únicamente se encontraron diferencias altamente significativas $(p<0,01)$ en número de rebrotes y número de ramas en botón de oro. El T4 obtuvo el mayor número de ramas: 127,10 y 819,65 rebrotes por arbusto (Cuadro 2).

Se analizaron los factores de forma independiente tanto para $T$. diversifolia como para $S$. nigra (Cuadro 3 y 4), en donde se identificó que el factor edad fue determinante, y que a mayor edad mayor producción de forraje.

La mayor producción de forraje verde se obtuvo a los 80 días en $T$. diversifolia con 4,14 kg/arbusto (Cuadro 3 ) y a los 90 días en $S$. nigra con $2,03 \mathrm{~kg}$ /arbusto (Cuadro 4). Se obtuvo mayor rendimiento en MS para ambas especies, las cuales mostraron mayor producción de forma significativa en el factor edad, con 0,68 kg/arbusto para T. diversifolia a los 80 días equivalente a $30,6 \mathrm{t} \mathrm{ha}^{-1} \mathrm{año}^{-1}$, y $0,36 \mathrm{~kg}$ /arbusto a los 90 días de corte en $S$. nigra, que se tradujo en un rendimiento de $14,4 \mathrm{t} \mathrm{ha}^{-1} \mathrm{año}^{-1}$. 


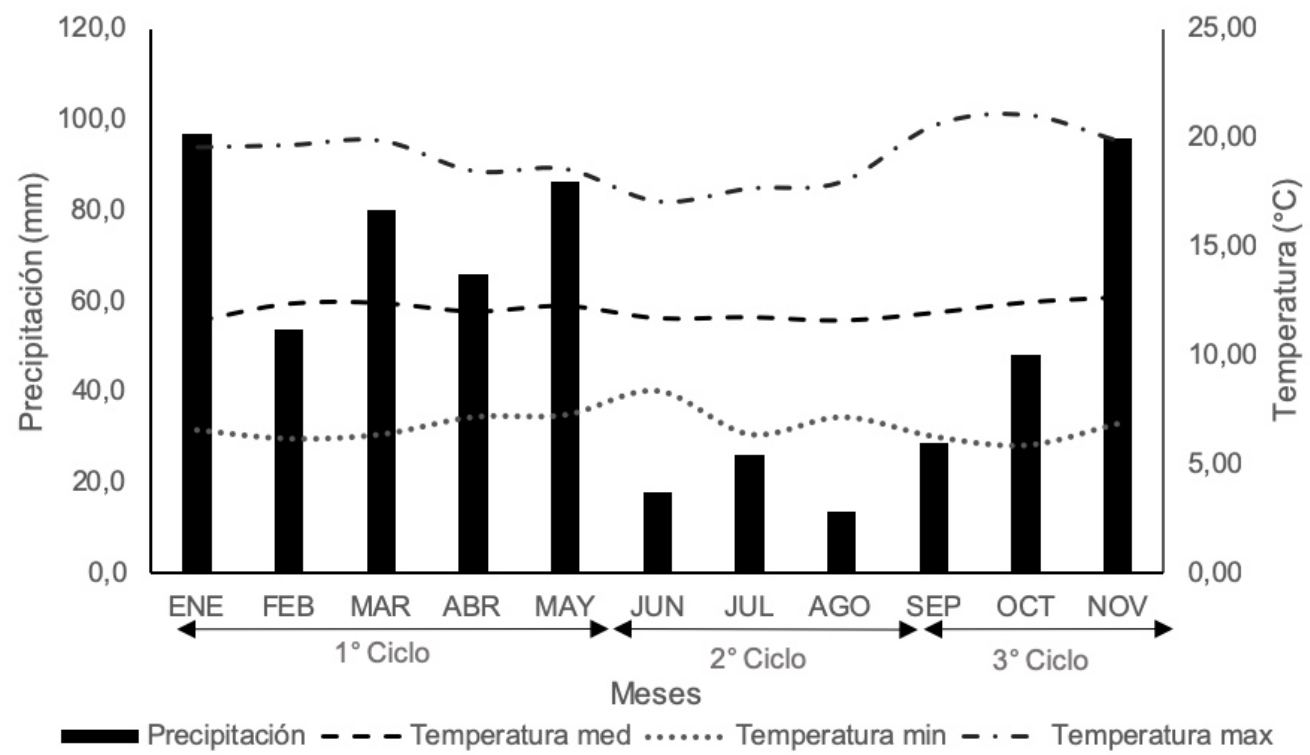

Figura 1. Distribución de la precipitación y temperatura durante tres ciclos de evaluación comprendidos entre enero a noviembre de 2018, Vintage station pro 2, ubicada en el centro de investigación Obonuco de la Corporación Colombiana de Investigación Agrícola (AGROSAVIA). Pasto, Colombia.

Figure 1. Precipitation and temperature distribution during three assessment cycles from January 2018 to November 2018, Vintage station pro 2, located in the Obonuco research center of the Corporación Colombiana de Investigación Agropecuaria (AGROSAVIA). Pasto, Colombia.

Cuadro 2. Evaluación de variables de rendimiento y morfométricas en tratamientos de T. diversifolia, en el Centro de Investigación Obonuco perteneciente a la Corporación Colombiana de Investigación Agropecuaria. Pasto, Colombia. 2019.

Table 2. Evaluation of yield and morphometric variables in treatments of T. diversifolia, in the Obonuco Research Center belonging to the Corporación Colombiana de Investigacion Agropecuaria (AGROSAVIA). Pasto, Colombia. 2019.

\begin{tabular}{|c|c|c|c|c|c|c|c|c|}
\hline \multicolumn{2}{|c|}{ Fuentes de variación } & \multirow{2}{*}{$\begin{array}{c}\begin{array}{c}\text { FV kg/ } \\
\text { arbusto }\end{array} \\
1,94^{\mathrm{a}}\end{array}$} & \multirow{2}{*}{$\begin{array}{c}\text { MS kg/ } \\
\text { arbusto }\end{array}$} & \multirow{2}{*}{$\begin{array}{c}\text { MS t ha-1 } \\
\text { año }^{-1} \\
15^{\mathrm{a}}\end{array}$} & \multirow{2}{*}{$\begin{array}{c}\begin{array}{c}\text { Diámetro de } \\
\text { copa }(\mathbf{c m})\end{array} \\
71,11^{\mathrm{a}}\end{array}$} & \multirow{2}{*}{$\begin{array}{c}\text { No. ramas } \\
35,46^{\mathrm{a}}\end{array}$} & \multirow{2}{*}{$\begin{array}{c}\begin{array}{c}\text { No. } \\
\text { rebrotes }\end{array} \\
198.66^{\mathrm{a}}\end{array}$} & \multirow{2}{*}{$\begin{array}{c}\text { No. botones } \\
0^{\mathrm{a}}\end{array}$} \\
\hline Edad & $\mathrm{T} 1$ & & & & & & & \\
\hline $\begin{array}{l}\text { de corte } \\
\text { (días)* }^{\text {de }}\end{array}$ & $\mathrm{T} 2$ & $1,95^{\mathrm{a}}$ & $0,26^{\mathrm{a}}$ & $11,17^{\mathrm{a}}$ & $75,29^{\mathrm{a}}$ & $49,23^{\mathrm{a}}$ & $256,02^{\mathrm{bc}}$ & $0,18^{\mathrm{a}}$ \\
\hline Altura de & $\mathrm{T} 3$ & $3,70^{\mathrm{a}}$ & $0,58^{\mathrm{a}}$ & $34,8^{\mathrm{a}}$ & $98,73^{\mathrm{a}}$ & $74,87^{b}$ & $424,89^{\mathrm{b}}$ & $0^{\mathrm{a}}$ \\
\hline corte $(\mathrm{cm})$ & $\mathrm{T} 4$ & $4,5^{\mathrm{a}}$ & $0,79^{\mathrm{a}}$ & $35,5^{\mathrm{a}}$ & $118,92^{\mathrm{a}}$ & $127,10^{\mathrm{c}}$ & $819,65^{\mathrm{c}}$ & $0,10^{\mathrm{a}}$ \\
\hline
\end{tabular}

a,b,c, medias con letras diferentes en las columnas difieren entre sí según la prueba de Tukey (p<0,05). T1:60 días*20 cm, T2:60 días*50 cm, T3:80 días*20 cm, T4:80 días $* 50 \mathrm{~cm} / \mathrm{a}, \mathrm{b}, \mathrm{c}$, means with different letters in the columns differ from each other according to the Tukey test $(\mathrm{p}<0.05)$. T1: 60 days $* 20 \mathrm{~cm}, \mathrm{~T} 2: 60$ days $* 50 \mathrm{~cm}, \mathrm{~T} 3: 80$ days $* 20 \mathrm{~cm}, \mathrm{~T} 4: 80$ days $* 50 \mathrm{~cm}$.

Con respecto a la variable diámetro de copa, los factores por separado presentaron diferencias significativas, donde se evidenció que para ambas especies se alcanzó un mayor diámetro de la copa cuando se cosechó a la mayor edad y altura evaluadas (Cuadros 3 y 4 ).

En la especie $S$. nigra, el número de ramas y rebrotes presentaron diferencias significativas $(\mathrm{p}<0,05)$ en los factores edad y altura de forma independiente, se evidenció que a mayor edad (90 días) hubo mayor número de 
Cuadro 3. Evaluación de variables de rendimiento y morfométricas en $T$. diversifolia en los factores independientes edad y altura de corte, en el Centro de Investigación Obonuco perteneciente a la Corporación Colombiana de Investigación Agropecuaria (AGROSAVIA). Pasto, Colombia. 2019.

Table 3. Evaluation of yield and morphometric variables in T. diversifolia in the independent factors age and height of cut in the Obonuco Research Center belonging to the Corporación Colombiana de Investigación Agropecuaria (AGROSAVIA). Pasto, Colombia. 2019.

\begin{tabular}{|c|c|c|c|c|c|c|c|c|}
\hline & & $\begin{array}{c}\text { FV kg/ } \\
\text { arbusto }\end{array}$ & $\begin{array}{l}\text { MS kg/ } \\
\text { arbusto }\end{array}$ & $\begin{array}{l}\text { MS t ha-1 } \\
\text { año-1 }^{-1}\end{array}$ & $\begin{array}{c}\text { Diámetro de } \\
\text { copa }(\mathbf{c m})\end{array}$ & No. ramas & $\begin{array}{c}\text { No. } \\
\text { rebrotes }\end{array}$ & No. botones \\
\hline \multirow{2}{*}{$\begin{array}{l}\text { Edad de } \\
\text { corte (días) }\end{array}$} & 60 & $1,94^{\mathrm{a}}$ & $0,26^{\mathrm{a}}$ & $15,6^{\mathrm{a}}$ & $84,92^{\mathrm{a}}$ & $55,16^{\mathrm{a}}$ & $311,78^{\mathrm{a}}$ & $0^{\mathbf{a}}$ \\
\hline & 80 & $4,14^{\mathrm{b}}$ & $0,68^{\mathrm{b}}$ & $30,6^{\mathrm{b}}$ & $96,82^{\mathrm{b}}$ & $87,66^{\mathrm{b}}$ & $534,18^{\mathrm{b}}$ & $0,14^{\mathrm{a}}$ \\
\hline \multirow{2}{*}{$\begin{array}{l}\text { Altura de } \\
\text { corte }(\mathrm{cm})\end{array}$} & 10 & $2,82^{\mathrm{a}}$ & $0,41^{\mathrm{a}}$ & $24,6^{\mathrm{a}}$ & $73,20^{\mathrm{a}}$ & $42,34^{\mathrm{a}}$ & $227,34^{\mathrm{a}}$ & $0,08^{\mathrm{a}}$ \\
\hline & 50 & $3,26^{\mathrm{a}}$ & $0,53^{\mathrm{a}}$ & $23,85^{\mathrm{a}}$ & $108,70^{\mathrm{b}}$ & $100,64^{\mathrm{b}}$ & $619,71^{\mathrm{b}}$ & $0,05^{\mathrm{a}}$ \\
\hline
\end{tabular}

a,b,c, medias con letras diferentes en las columnas difieren entre sí según la prueba de Tukey $(\mathrm{p}<0,05)$ / a, b, c, means with different letters in the columns differ from each other according to the Tukey test $(\mathrm{p}<0.05)$.

Cuadro 4. Evaluación de variables de rendimiento y morfométricas en S. nigra en los factores independientes edad y altura de corte en el Centro de Investigación Obonuco perteneciente a la Corporación Colombiana de Investigación Agropecuaria (AGROSAVIA). Pasto, Colombia. 2019.

Table 4. Evaluation of yield and morphometric variables in S. nigra in the independent factors age and cutting height in the Obonuco Research Center belonging to the Corporación Colombiana de Investigación Agropecuaria (AGROSAVIA). Pasto, Colombia. 2019.

\begin{tabular}{|c|c|c|c|c|c|c|c|c|}
\hline & & $\begin{array}{c}\text { FV kg/ } \\
\text { arbusto }\end{array}$ & $\begin{array}{l}\text { MS kg/ } \\
\text { arbusto }\end{array}$ & $\begin{array}{l}\text { MS t ha-1 } \\
\text { año }^{-1}\end{array}$ & $\begin{array}{l}\text { Diámetro de } \\
\text { copa }(\mathrm{cm})\end{array}$ & No. ramas & $\begin{array}{c}\text { No. } \\
\text { rebrotes }\end{array}$ & $\begin{array}{c}\text { No. } \\
\text { botones }\end{array}$ \\
\hline \multirow{2}{*}{$\begin{array}{l}\text { Edad de } \\
\text { corte (días) }\end{array}$} & 60 & $0,92^{\mathrm{a}}$ & $0,18^{\mathrm{a}}$ & $10,8^{a}$ & $58,30^{\mathrm{a}}$ & $36,90^{\mathrm{a}}$ & $145,25^{\mathrm{a}}$ & $0,12^{\mathrm{a}}$ \\
\hline & 90 & $2,03^{\mathrm{b}}$ & $0,36^{\mathrm{b}}$ & $14,4^{\mathrm{b}}$ & $66,24^{\mathrm{b}}$ & $54,11^{\mathrm{b}}$ & $262,16^{\mathrm{b}}$ & $0,70^{\mathrm{b}}$ \\
\hline \multirow{2}{*}{$\begin{array}{l}\text { Altura de } \\
\text { corte }(\mathrm{cm})\end{array}$} & 30 & $1,38^{\mathrm{a}}$ & $0,25^{\mathrm{a}}$ & $10^{\mathrm{a}}$ & $55,76^{\mathrm{a}}$ & $39,02^{\mathrm{a}}$ & $161,63^{\mathrm{a}}$ & $0,38^{\mathrm{a}}$ \\
\hline & 50 & $1,57^{\mathrm{a}}$ & $0,29^{\mathrm{a}}$ & $11,6^{\mathrm{a}}$ & $68,79^{\mathrm{b}}$ & $51,98^{\mathrm{b}}$ & $245,77^{\mathrm{b}}$ & $0,44^{\mathrm{a}}$ \\
\hline
\end{tabular}

a,b,c, medias con letras diferentes en las columnas difieren entre sí según la prueba de Tukey $(\mathrm{p}<0,05)$ / a, b, c, means with different letters in the columns differ from each other according to the Tukey test $(\mathrm{p}<0.05)$.

ramas (54) y de rebrotes (262). En cuanto al factor altura de corte, se identificó que a mayor altura $(50 \mathrm{~cm})$ hubo mayor número de ramas $(51,98)$ y de rebrotes $(245,77)$ (Cuadro 4). Finalmente, la variable número de botones mostró significancia en el factor edad, evidenciando que fue superior en los arbustos cortados a los 90 días en $S$. nigra, mientras que en botón de oro no presentó significancia.

\section{Vigor}

$\mathrm{Al}$ analizar el vigor por medio de la prueba Chi Cuadrado $(\mathrm{p}<0,05)$, se encontraron diferencias significativas en ambas especies. En el caso de T. diversifolia se observó que los tratamientos T2 y T4 (T2: 60 días-50 cm de altura de corte; T4: 80 días-50 cm de altura de corte) presentaron la mayor cantidad de individuos con vigor denominado como: muy bueno (29,2\% y $26,4 \%$, respectivamente). En la especie $S$. nigra, se encontraron resultados similares, sobresaliendo los tratamientos 2 y 4 (T2: 60 días-50 cm de altura de corte; T4: 90 días-50 cm de altura de corte) con porcentajes de 31,9 y 27,8 , respectivamente. De acuerdo con los resultados obtenidos, las dos especies arbustivas llegaron a tener un buen comportamiento en cuanto a vigor cuando se cosecharon a mayor altura, en este caso 50 
cm del suelo, independiente de las edades; sin embargo, cabe resaltar que a los 60 días se obtuvo un mejor vigor en ambas especies.

\section{Composición química de forraje}

Los valores para calidad composicional derivados del análisis bromatológico en el forraje de $T$. diversifolia presentaron diferencias significativas $(\mathrm{p}<0,05)$ en la interacción edad*altura para las variables PC, FDA, NDT, digestibilidad y ENL (Cuadro 5), igualmente en la especie $S$. nigra, a excepción de FDA que no presentó diferencia significativa $(\mathrm{p} \geq 0,05)$ (Cuadro 6). Las diferencias estuvieron asociadas principalmente al factor edad, los valores en T1 y T2 (correspondientes a edad de 60 días en T. diversifolia y $S$. nigra) fueron diferentes a T3 y T4 (correspondientes a edad de 80 días en T. diversifolia y 90 días en $S$. nigra). Por lo tanto, se analizó el factor independiente edad en ambas especies, en donde fue más evidente el efecto significativo $(\mathrm{p}<0,05)$ sobre las variables de calidad composicional.

Cuadro 5. Composición química de la especie T. diversifolia en la interacción edad*altura de corte, en el Centro de Investigación Obonuco, Corporación Colombiana de Investigación Agropecuaria (AGROSAVIA). Pasto, Colombia. 2019.

Table 5. Chemical composition of the T. diversifolia species in the age * cutting height interaction , in the Obonuco Research Center belonging to the Corporación Colombiana de Investigación Agropecuaria (AGROSAVIA). Pasto, Colombia. 2019.

\begin{tabular}{cccccccccccc}
\hline $\begin{array}{c}\text { Fuentes de } \\
\text { variación }\end{array}$ & $\begin{array}{c}\text { PC } \\
(\boldsymbol{\%})\end{array}$ & $\begin{array}{c}\text { FDN } \\
(\boldsymbol{\%})\end{array}$ & $\begin{array}{c}\text { FDA } \\
(\boldsymbol{\%})\end{array}$ & $\begin{array}{c}\text { Lig. } \\
(\boldsymbol{\%})\end{array}$ & $\begin{array}{c}\text { Hem. } \\
(\boldsymbol{\%})\end{array}$ & $\begin{array}{c}\text { Ca } \\
\boldsymbol{\%})\end{array}$ & $\begin{array}{c}\text { P } \\
(\boldsymbol{\%})\end{array}$ & $\begin{array}{c}\text { NDT } \\
(\boldsymbol{\%})\end{array}$ & $\begin{array}{c}\text { Dig. } \\
(\boldsymbol{\%})\end{array}$ & $\begin{array}{c}\text { ENL (Mcal/ } \\
\text { kg MS) }\end{array}$ \\
\hline $\begin{array}{c}\text { Edad } \\
(\text { días) }\end{array}$ & $\mathrm{T} 1$ & $26,51^{\mathrm{a}}$ & $32,77^{\mathrm{a}}$ & $14,84^{\mathrm{b}}$ & $2,76^{\mathrm{a}}$ & $19,96^{\mathrm{a}}$ & $0,88^{\mathrm{a}}$ & $0,30^{\mathrm{a}}$ & $68,98^{\mathrm{a}}$ & $75,32^{\mathrm{a}}$ & $1,57^{\mathrm{a}}$ \\
\multirow{2}{*}{$\begin{array}{c}\text { Altura de } \\
\text { corte }(\mathbf{c m})\end{array}$} & $\mathrm{T} 2$ & $26,96^{\mathrm{a}}$ & $33,35^{\mathrm{a}}$ & $15,21^{\text {ab }}$ & $2,77^{\mathrm{a}}$ & $20,48^{\mathrm{a}}$ & $0,86^{\mathrm{a}}$ & $0,31^{\mathrm{a}}$ & $69,22^{\mathrm{a}}$ & $75,57^{\mathrm{a}}$ & $1,58^{\mathrm{a}}$ \\
& $\mathrm{T} 3$ & $21,44^{\mathrm{b}}$ & $34,44^{\mathrm{a}}$ & $17,03^{\mathrm{ab}}$ & $2,96^{\mathrm{a}}$ & $15,38^{\mathrm{a}}$ & $0,77^{\mathrm{a}}$ & $0,29^{\mathrm{a}}$ & $65,78^{\mathrm{b}}$ & $71,87^{\mathrm{b}}$ & $1,50^{\mathrm{b}}$ \\
\hline
\end{tabular}

a,b,c, medias con letras diferentes en las columnas difieren entre sí según la prueba de Tukey (p<0,05). T1:60 días*20 cm, T2:60 días*50 $\mathrm{cm}$, T3:80 días*20 cm, T4:80 días*50 cm / a,b,c, averages with different letters in the columns differ from each other according to Tukey's test $(\mathrm{p}<0.05)$. T1:60 days $* 20 \mathrm{~cm}, \mathrm{~T} 2: 60$ days $* 50 \mathrm{~cm}, \mathrm{~T} 3: 80$ days $* 20 \mathrm{~cm}, \mathrm{~T} 4: 80$ days*50 cm.

Cuadro 6. Composición química de la especie S. nigra en la interacción edad*altura de corte, en el Centro de Investigación Obonuco perteneciente a la Corporación Colombiana de Investigación Agropecuaria (AGROSAVIA). Pasto, Colombia. 2019.

Table 6. Chemical composition of the S. nigra species in the age* cutting height interaction, in the Obonuco Research Center belonging to the Corporación Colombiana de Investigación Agropecuaria (AGROSAVIA). Pasto, Colombia. 2019.

\begin{tabular}{|c|c|c|c|c|c|c|c|c|c|c|c|}
\hline \multicolumn{2}{|c|}{$\begin{array}{c}\text { Fuentes de } \\
\text { variación }\end{array}$} & $\begin{array}{l}\text { PC } \\
(\%)\end{array}$ & $\begin{array}{c}\text { FDN } \\
(\%)\end{array}$ & $\begin{array}{c}\text { FDA } \\
(\%)\end{array}$ & $\begin{array}{l}\text { Lig. } \\
(\%)\end{array}$ & $\begin{array}{c}\text { Hem. } \\
(\%)\end{array}$ & $\begin{array}{l}\mathrm{Ca} \\
(\%)\end{array}$ & $\begin{array}{c}\mathbf{P} \\
(\%)\end{array}$ & $\begin{array}{l}\text { NDT } \\
(\%)\end{array}$ & $\begin{array}{l}\text { Dig. } \\
(\%)\end{array}$ & $\begin{array}{c}\text { ENL (Mcal. } \\
\text { kg }^{-1} \text { MS) }\end{array}$ \\
\hline \multirow{4}{*}{$\begin{array}{c}\text { Edad } \\
\text { (días)* } \\
\text { Altura } \\
\text { de corte } \\
\text { (cm) }\end{array}$} & T1 & $26,50^{\mathrm{a}}$ & $27,72^{\mathrm{a}}$ & $10,93^{\mathrm{a}}$ & $1,80^{\mathrm{a}}$ & $16,49^{\mathrm{a}}$ & $0,82^{\mathrm{a}}$ & $0,24^{a}$ & $70,01^{\mathrm{a}}$ & $76,43^{\mathrm{a}}$ & $1,60^{\mathrm{a}}$ \\
\hline & $\mathrm{T} 2$ & $26,01^{\mathrm{a}}$ & $28,86^{a}$ & $11,44^{\mathrm{a}}$ & $2,01^{\mathrm{a}}$ & $16,59^{\mathrm{a}}$ & $0,81^{\mathrm{a}}$ & $0,23^{\mathrm{a}}$ & $69,73^{\mathrm{a}}$ & $76,13^{\mathrm{a}}$ & $1,59^{\mathrm{a}}$ \\
\hline & T3 & $20,93^{b}$ & $28.57^{\mathrm{a}}$ & $14,06^{\mathrm{a}}$ & $2,49^{\mathrm{a}}$ & $14,82^{\mathrm{a}}$ & $0,83^{\mathrm{a}}$ & $0,21^{\mathrm{a}}$ & $66,38^{b}$ & $72,52^{\mathrm{b}}$ & $1,51^{\mathrm{b}}$ \\
\hline & $\mathrm{T} 4$ & $21,10^{\mathrm{b}}$ & $28.49^{\mathrm{a}}$ & $13,94^{\mathrm{a}}$ & $2,54^{\mathrm{a}}$ & $14,51^{\mathrm{a}}$ & $0,84^{a}$ & $0,21^{\mathrm{a}}$ & $66,42^{\mathrm{b}}$ & $72,56^{\mathrm{b}}$ & $1,51^{\mathrm{b}}$ \\
\hline
\end{tabular}

a,b,c, medias con letras diferentes en las columnas difieren entre sí según la prueba de Tukey (p<0,05). T1: 60 días*30 cm, T2: 60 días*50 cm, T3: 90 días*30 cm, T4: 90 días*50 cm / a,b,c, averages with different letters in the columns differ from each other according to Tukey's test $(\mathrm{p}<0.05)$. T1: 60 days $* 30 \mathrm{~cm}, \mathrm{~T} 2: 60$ days $* 50 \mathrm{~cm}, \mathrm{~T} 3: 90$ days $* 30 \mathrm{~cm}, \mathrm{~T} 4: 90$ days $* 50 \mathrm{~cm}$. 
La composición química del forraje en $T$. diversifolia a los 60 y 80 días de corte (Cuadro 7) presentó diferencias significativas en ocho de las variables evaluadas, correspondientes a proteína cruda $(26,73 \%$ vs 21,37 $\%$ respectivamente), fibra en detergente ácido $(15,02 \%$ vs $17,35 \%)$, hemicelulosa $(20,22 \%$ vs $15,55 \%)$, calcio $(0,87 \%$ vs $0,77 \%)$, fósforo $(0,31 \%$ vs $0,29 \%)$, nutrientes digestibles totales $(69,10 \%$ vs $65,63 \%)$, digestibilidad in vitro de la MS (75,45\% vs 71,71\%) y energía neta de lactancia (1,58 Mcal kg-1 MS vs 1,49 $\left.\mathrm{Mcal} \mathrm{kg}^{-1} \mathrm{MS}\right)$, evidenciando mejores valores a edad de 60 días.

Cuadro 7. Evaluación de variables de calidad para T. diversifolia en los factores independientes edad y altura de corte en el Centro de Investigación Obonuco, Corporación Colombiana de Investigación Agropecuaria (AGROSAVIA). Pasto, Colombia. 2019.

Table 7. Evaluation of quality variables for T. diversifolia in the independent factors age and cutting height in the Obonuco Research Center belonging to the Corporación Colombiana de Investigación Agropecuaria (AGROSAVIA). Pasto, Colombia. 2019.

\begin{tabular}{|c|c|c|c|c|c|c|c|c|c|c|c|}
\hline & & PC (\%) & $\begin{array}{l}\text { FDN } \\
(\%)\end{array}$ & $\begin{array}{l}\text { FDA } \\
(\%)\end{array}$ & Lig. (\%) & $\begin{array}{c}\text { Hem. } \\
(\%)\end{array}$ & Ca (\%) & $\mathbf{P}(\%)$ & $\begin{array}{l}\text { NDT } \\
(\%)\end{array}$ & Dig. (\%) & $\begin{array}{c}\text { ENL (Mcal. } \\
\mathrm{kg}^{-1} \mathrm{MS} \text { ) }\end{array}$ \\
\hline \multirow{2}{*}{ Edad (días) } & 60 & 26 & $33,06^{\mathrm{a}}$ & $15,02^{\mathrm{b}}$ & $2,77^{\mathrm{a}}$ & $20,22^{\mathrm{a}}$ & $0,87^{\mathrm{a}}$ & $\mathbf{0 , 3 1 ^ { \mathrm { a } }}$ & $69,10^{\mathrm{a}}$ & $75,45^{\mathrm{a}}$ & $1,58^{\mathrm{a}}$ \\
\hline & 80 & $21,37^{b}$ & $35,09^{\mathrm{a}}$ & $17,35^{\mathrm{a}}$ & $3,01^{\mathrm{a}}$ & $15,55^{\mathrm{b}}$ & $0,77^{\mathrm{b}}$ & $0,29^{b}$ & $65,63^{\mathrm{b}}$ & $71,71^{\mathrm{b}}$ & $1,49^{\mathrm{b}}$ \\
\hline \multirow{2}{*}{$\begin{array}{l}\text { Altura de } \\
\text { corte }(\mathrm{cm})\end{array}$} & 10 & 23,9 & $33,60^{\mathrm{a}}$ & $15,93^{\mathrm{a}}$ & $2,86^{\mathrm{a}}$ & 7a & 0,8 & 0,3 & $67,38^{a}$ & $73,60^{a}$ & $1,54^{\mathrm{a}}$ \\
\hline & 50 & $24,13^{\mathrm{a}}$ & $34,54^{\mathrm{a}}$ & $16,45^{\mathrm{a}}$ & $2,92^{\mathrm{a}}$ & $18,10^{\mathrm{a}}$ & $0,81^{\mathrm{a}}$ & $0,30^{\mathrm{a}}$ & $67,35^{\mathrm{a}}$ & $73,56^{\mathrm{a}}$ & $1,53^{\mathrm{a}}$ \\
\hline
\end{tabular}

a,b,c, medias con letras diferentes en las columnas difieren entre sí según la prueba de Tukey $(\mathrm{p}<0,05)$ / a,b,c, averages with different letters in the columns differ from each other according to Tukey's test $(\mathrm{p}<0.05)$.

En S. nigra se presentó efecto de la edad de corte (60 y 90 días) en siete de las variables evaluadas (Cuadro 8), correspondientes a proteína cruda $(26,25 \%$ vs $21,01 \%)$, lignina $(1,91 \%$ vs $2,52 \%)$, hemicelulosa ( $16,54 \%$ vs $14,67 \%)$, fósforo $(0,24 \%$ vs $0,21 \%)$, nutrientes digestibles totales $(69,88 \%$ vs $66,40 \%)$, digestibilidad in vitro MS (76,28 \% vs 72,54\%) y energía neta de lactancia (ENL) (1,60 Mcal kg ${ }^{-1} \mathrm{MS}$ vs $\left.1,51 \mathrm{Mcal} \mathrm{kg} \mathrm{MS}^{-1}\right)$; con un comportamiento similar a $T$. diversifolia con mejores valores a 60 días de corte.

Cuadro 8. Evaluación de variables de vigor para T. diversifolia en los factores independientes edad y altura de corte en el Centro de Investigación Obonuco, Corporación Colombiana de Investigación Agropecuaria (AGROSAVIA). Pasto, Colombia. 2019.

Table 8. Evaluation of igor variables for T. diversifolia in the independent factors age and cutting height in the Obonuco Research Center belonging to the Corporación Colombiana de Investigación Agropecuaria (AGROSAVIA). Pasto, Colombia. 2019.

\begin{tabular}{|c|c|c|c|c|c|c|c|c|c|c|c|}
\hline & & PC (\%) & $\begin{array}{l}\text { FDN } \\
(\%)\end{array}$ & $\begin{array}{l}\text { FDA } \\
(\%)\end{array}$ & Lig. (\%) & $\begin{array}{c}\text { Hem. } \\
(\%)\end{array}$ & Ca (\%) & $\mathbf{P}(\%)$ & $\begin{array}{l}\text { NDT } \\
(\%)\end{array}$ & Dig. (\%) & $\begin{array}{c}\text { ENL (Mcal. } \\
\mathrm{kg}^{-1} \mathrm{MS} \text { ) }\end{array}$ \\
\hline \multirow{2}{*}{$\begin{array}{c}\text { Edad de } \\
\text { corte (días) }\end{array}$} & 60 & $25^{\mathrm{a}}$ & $28,29^{\mathrm{a}}$ & $11,18^{b}$ & $1,91^{\mathrm{b}}$ & $16,54^{\mathrm{a}}$ & $0,81^{\mathrm{a}}$ & & $69,88^{a}$ & $8^{a}$ & $1,60^{\mathrm{a}}$ \\
\hline & 90 & $21,01^{\mathrm{b}}$ & $28,53^{a}$ & $13,99^{a}$ & $2,52^{\mathrm{a}}$ & $14,67^{\mathrm{b}}$ & $0,83^{\mathrm{a}}$ & $0,21^{\mathrm{b}}$ & $66,40^{\mathrm{b}}$ & $72,54^{\mathrm{b}}$ & $1,51^{\mathrm{b}}$ \\
\hline \multirow{2}{*}{$\begin{array}{l}\text { Altura de } \\
\text { corte }(\mathrm{cm})\end{array}$} & 30 & $23,71^{a}$ & $28,15^{\mathrm{a}}$ & 12 , & 2,1 & 15 & 0,8 & 0,2 & $68,19^{\mathrm{a}}$ & $74,47^{a}$ & $1,55^{\mathrm{a}}$ \\
\hline & 50 & $23,56^{a}$ & $28,68^{\mathrm{a}}$ & $12,68^{\mathrm{a}}$ & $2,28^{\mathrm{a}}$ & $15,55^{\mathrm{a}}$ & $0,82^{\mathrm{a}}$ & $0,22^{\mathrm{a}}$ & $68,08^{a}$ & $74,35^{\mathrm{a}}$ & $1,55^{\mathrm{a}}$ \\
\hline
\end{tabular}

a,b,c, medias con letras diferentes en las columnas difieren entre sí según la prueba de Tukey $(\mathrm{p}<0,05)$ / a,b,c, averages with different letters in the columns differ from each other according to Tukey's test $(\mathrm{p}<0.05)$. 


\section{Discusión}

Los resultados de biomasa para $T$. diversifolia obtenidos en la presente investigación fueron superiores a los 80 días de corte $\left(30,6 \mathrm{t} \mathrm{ha}^{-1}\right.$ año-1), lo cual coincide con las cifras reportadas por Navarro y Rodríguez (1990), quienes encontraron una producción de MS a los 74 días de 30,15 $\mathrm{t} \mathrm{ha}^{-1} \mathrm{año}^{-1} \mathrm{vs} 17,25 \mathrm{t} \mathrm{ha}^{-1}$ año $\mathrm{a}^{-1}$ a los 60 días. En un estudio sobre el efecto de la distancia de siembra $(0,5 \mathrm{~m}-1 \mathrm{~m})$, frecuencia (40, 60 y 80 días) y altura de corte $(5,10$ y 15 $\mathrm{cm}$ ), se encontró la mayor producción de $T$. diversifolia a $0,5 \mathrm{~m}$ de distancia de siembra, 80 días de corte y $10 \mathrm{~cm}$ de altura (0,85-0,90 t ha-1 MS) (Ruiz et al., 2012), lo cual muestra una tendencia al aumento de la materia seca cuando la edad es mayor. Por el contrario, al estudiar el efecto de la frecuencia de poda (cada 2, 4 y 8 semanas) se encontró la mayor producción de materia seca a las 8 semanas (60 días) a $50 \mathrm{~cm}$ de altura de corte con un rendimiento de $25,8 \mathrm{t} \mathrm{ha}^{-1} \mathrm{año}^{-1}$ (Partey, 2011). Esta diferencia se puede atribuir posiblemente a las diferencias de altitud en los estudios realizados, ya que de acuerdo con Zapata y Vargas (2014), a menor altitud, mayor radiación; por lo tanto la tasa fotosintética es mayor y se produce más forraje.

En cuanto al rendimiento de MS en sauco, Apráez et al. (2012), reportaron a edad de 90 días una producción de 19,6\% equivalente a 15,2 tha año $^{-1}$, lo cual concuerda con el encontrado en el presente estudio a la misma edad $\left(14,4\right.$ t ha $^{-1}$ año $\left.^{-1}\right)$. Por otra parte, Cárdenas et al. (2016) reportaron el efecto de cuatro edades de corte $(90,120,150$ y 180 días) sobre la biomasa de sauco y registraron un rendimiento de $12,72 \mathrm{t} \mathrm{ha}^{-1}$ año $^{-1}(0,318 \mathrm{~kg} /$ arbusto $)$ a los 90 días, valor menor al encontrado en el presente estudio para el mismo plazo; estos autores señalaron que la mejor edad de corte es a los 180 días, ya que registran el rendimiento más alto con $32,4 \mathrm{t} \mathrm{ha}^{-1} \mathrm{año}^{-1}$ de MS (2,3 $\mathrm{kg}$ por arbusto). El comportamiento en las variables forraje verde y MS de la especie $S$. nigra, estuvieron influenciados por la edad, de modo que a mayor edad (90 días), mayor producción, ya que al prolongarse el tiempo de recuperación, se generó mayor cantidad de follaje.

En cuanto a la variable diámetro de copa, los resultados indicaron que en botón de oro y sauco se presentó tendencia a obtener un diámetro más amplio cuando se cosecharon a mayor edad (80 días - 90 días respectivamente) y altura $(50 \mathrm{~cm})$; lo cual concuerda con un estudio reportado en sauco, donde el diámetro de copa obtuvo una mayor medida al aumentar la edad de corte en evaluación, que correspondía a 180 días, obteniendo un diámetro de 168 cm vs $74 \mathrm{~cm}$ valor obtenido a los 90 días (Cárdenas et al., 2016). A pesar de ser valores superiores al encontrado en esta investigación, se demuestra la tendencia al aumento del diámetro cuando incrementa la edad.

Con respecto al número de ramas y rebrotes en la especie botón de oro, se presentaron diferencias en los tratamientos. El T4 obtuvo la mayor cantidad de ramas, esto posiblemente se presentó porque al cosechar la planta a $50 \mathrm{~cm}$ del suelo quedaron mayor cantidad de tallos, lo cual garantiza en las plantas la presencia de un área adecuada de los tejidos parenquimático de reserva y meristemático activo, ambos factores necesarios en el desarrollo del rebrote (Stür et al., 1994). Al cosechar a una edad mayor (80 días en este estudio), se espera que una alta cantidad de yemas se desarrollen y se conviertan en tallos, lo cual se refleja en una mayor producción de biomasa. Esta tendencia, también la reportaron Ella et al. (1991), quienes ratificaron que las plantas de mayor edad presentaron un mayor número de ramificaciones como consecuencia de una elevada cantidad de puntos de crecimiento y de reservas, las cuales son responsables de un mayor número de rebrotes, independientemente de las hojas remanentes en las plantas después del corte o el pastoreo.

Lo anterior corrobora el comportamiento encontrado en ambas especies en la variable vigor, ya que se identificó que a mayor edad y altura de corte se presentó el mejor vigor; resultados que reiteran el potencial forrajero de $T$. diversifolia y $S$. nigra, el cual está ligado directamente a su tolerancia a la poda y capacidad de rebrote, efecto que permite obtener gran cantidad de biomasa por unidad de área (Sánchez et al., 2010; Lugo et al., 2012).

Con respecto a la variable número de botones se evidencio que a mayor edad mayor aparición de botones florales (90 días en este estudio), lo cual es un resultado esperado; ya que a medida que aumentó la edad existió más probabilidad de generar los botones florales, como un proceso natural de las etapas fenológicas de los cultivos. Para 
el botón de oro, Calle y Murgueitio (2014) recomendaron efectuar la cosecha antes de la floración, aproximadamente cada 50 días; mientras que Parada (2006) recomendó efectuar el corte de T. diversifolia cuando las plantas alcanzan la etapa de botones florales ( $75 \%$ botones por planta), que coincide con la presentación de la primera flor. Esta variable es determinante para definir la edad de corte que garantice una mejor calidad nutricional, ya que, con el incremento de la edad, aumenta el porcentaje de tallos, con la consiguiente disminución de la digestibilidad y el porcentaje de hojas, reduciendo gradualmente el contenido nutricional de la planta (Bragachini et al., 2008).

Los resultados en cuanto a PC encontrados en la presente investigación para el forraje de $T$. diversifolia, bajo diferentes edades de corte, contrastaron con el comportamiento reportado por Navarro y Rodríguez (1990) y Lezcano et al. (2012), quienes al evaluar la calidad de forraje de T. diversifolia a $1285 \mathrm{msnm}$ y $19 \mathrm{msnm}$ encontraron que la PC fue mayor a los treinta días (28,8\% y 29,79\%, respectivamente), mientras que a los 60 días fue de $22 \%$ y $28,69 \%$, respectivamente. La edad de corte de 60 días, fue considerada como una etapa de floración media (Navarro y Rodríguez, 1990); sin embargo, en el presente estudio a 2750 msnm, la misma edad correspondió a etapa de prefloración y reflejó el mayor valor para PC (26,73\%), lo anterior posiblemente se debió a que a mayor altura la floración es más lenta. Estudios han mostrado 15,85 \% de PC a 60 días de emergencia (Sanabria y Ávila, 2015) y 14,1 \% a los 56 días del corte de uniformización en plántulas de $T$. diversifolia propagadas por estaca (Gallego et al., 2017). A partir de lo anterior, se podría inferir que es importante manejar un tiempo adecuado de establecimiento de la especie para aprovechar su potencial nutricional, en este estudio se evaluó la edad de corte a 60 días en arbustos que tenían año y medio de establecidos y el valor de proteína cruda (26,73\%) mejoró considerablemente con respecto a las citadas investigaciones.

El mejor nivel de proteína en S. nigra se encontró a los 60 días $(26,25 \%)$, y fue mayor que el reportado para trópico de altura oscilante entre 15,22 y 25,2 \% (Millán y Moreno, 2005; Jaramillo, 2006; Cárdenas et al., 2011; Carvajal y Cuesta, 2016; Cortés y Ramos, 2018). En reportes de proteína cruda para S. nigra, se encontró que el mayor porcentaje se presentó a los 90 días, edad de corte que correspondía a la mínima evaluada por los autores. Sin embargo, en la presente investigación se encontró mayor porcentaje de PC a una edad menor (60 días), de igual manera ambos estudios concuerdan en que a mayores edades de corte, la proteína cruda del forraje tendió a disminuir en el trópico de altura. En este sentido, Barreto y Chamorro (2005), observaron a los 40, 60, 70 y 80 días de corte valores de 30,2\%; 30,9\%; 29,4\% y 29,6\% de proteína, respectivamente, disminuyendo un 2,05\% el contenido de esta. El valor nutritivo del forraje de árboles disminuye a medida que incrementa la madurez de hojas y tallos, ya que al cosechar la planta en estadios avanzados de maduración se presenta mayor proporción de fibra y lignina, pero más baja energía (Blanco y Sierra, 2005). De acuerdo con lo reportado por Carvajal et al. (2012), los valores de proteína cruda encontrados en el presente estudio $(26,73 \%)$ equivaldrían e incluso superarían a la fracción de PC que contiene un concentrado comercial $(18,0 \%)$.

Estimaciones del porcentaje de FDA en T. diversifolia que contrastan con los encontrados en esta investigación a 2750 msnm tanto a los 60 como 80 días de corte (15,02 y 17,35\% respectivamente), corresponden a los reportados por Téllez y Mendoza (2014) y Molina (2016) a 45 días de rebrote en elevaciones entre los 321 y 350 msnm, con porcentajes de 42,2 y 39,02. Según un estudio, estas variaciones en los porcentajes de FDA pueden deberse a las condiciones edafoclimáticas (Bernal, 2003), por lo tanto, este comportamiento se puede relacionar con las diferencias altitudinales de las zonas en las cuales se llevó a cabo las respectivas investigaciones. En el trópico bajo los procesos fisiológicos de la planta ocurren más rápidamente, relacionados sobre todo con el aumento de la temperatura (Rawson y Gómez, 2001); por ende, los procesos de maduración de las plantas asociados al incremento de los porcentajes de fibra se aceleran a menores altitudes sobre el nivel del mar.

Los valores de FDA evidenciados en esta investigación para $S$. nigra a los 60 y 90 días (11,18 y 15,99\%, respectivamente), fueron inferiores a los reportados por Jaramillo y Jiménez (2000) y Apráez et al. (2012) a 90 días de corte con 17,28 y 15,8\%, respectivamente. De igual manera, García y Ortegón (2014) y Carvajal et al. (2012), a 63 días de corte, reportaron porcentajes mayores con 16,67 \% y 15,72 \%, respectivamente. La baja proporción 
de FDA encontrada en este estudio podría relacionarse con la digestibilidad del forraje, ya que esta fue mayor a 60 días de edad de corte (76,28 \%), valor relativamente similar al 75,8 \% reportado por Muhammad et al. (1999).

En T. diversifolia la disminución del contenido de minerales a medida que se desarrollaba la planta fue evidente al evaluar 60 y 80 días de corte $(0,87 \%$ vs $0,77 \%$ en Ca y $0,31 \%$ vs $0,29 \%$ en P). Comportamiento similar reportaron Navarro y Rodríguez (1990) en Ibagué Tolima, con valores de 2,25 \% (30 días) a 1,65 \% (89 días) para el calcio y de 0,39 \% (30 días) a 0,32 \% (89 días) para el fósforo. Por su parte, Gallego-Castro et al. (2017) en el municipio de Guarne, Antioquia a $2453 \mathrm{msnm}$, reportaron a los 56 días de corte valores de calcio de 2,86 \% y fósforo de $0,27 \%$, y Cardona-Iglesias et al. (2017), en un estudio realizado a $2500 \mathrm{msnm}$ en el departamento de Antioquia (Colombia), encontraron un contenido superior de Ca y $\mathrm{P}(0,8$ y $0,35 \%)$ en plantas de Tithonia diversifolia a 70 días de cosecha, establecidas en sistema silvopastoril (SSP), respecto a pasturas de kikuyo (Cenchrus clandestinus Hochst. ex Chiov) de 35 días, asociada a dicho SSP (0,4 \% Ca y 0,2 \% P). Respecto a lo anterior, el contenido de fósforo en $T$. diversifolia se puede considerar como alto comparado con otras especies que se usan comúnmente en la alimentación del ganado (Mahecha y Rosales, 2005).

Se reportó 75,96\% de NDT a 60 días de germinación de plántulas de T. diversifolia, valor mayor al encontrado en el presente estudio $(69,1 \%)$, en el cual las plantas evaluadas tenían año y medio de establecimiento; por ende, tenían más desarrolladas estructuras como tallos que, al acumular contenido fibroso, reducen la digestibilidad del forraje. Sin embargo, el valor de NDT reportado en esta investigación es relativamente alto y se relaciona con los buenos contenidos de digestibilidad encontrados a 60 días en la literatura $(75,45 \%)$ (Sanabria y Ávila, 2015).

La digestibilidad del forraje de T. diversifolia en la presente investigación a 60 días de corte $(75,45 \%)$, fue superior al reportado por Téllez y Mendoza (2014) a $321 \mathrm{msnm}$, con porcentaje de 57,6 a edad de 45 días; evidenciando que muy posiblemente en el forraje cosechado a 60 días, la cantidad de fracciones alimenticias serán altamente aprovechadas por el animal. La diferencia entre estas digestibilidades, puede estar asociada a que las temperaturas del lugar en donde se llevó a cabo el presente experimento a $2750 \mathrm{msnm}$, las cuales fueron en promedio de $12,8^{\circ} \mathrm{C}$; mientras que la investigación de Téllez y Mendoza (2014) se desarrolló en clima más cálido; y la digestibilidad disminuye como resultado de la combinación de dos efectos de la alta temperatura ambiental: aumento de la lignificación de la pared celular, e incremento de la actividad metabólica (Van-Soest, 1994).

En la especie S. nigra Grajales et al. (2015), recomendaron efectuar el corte del forraje entre los 60 y 80 días, para garantizar la buena calidad nutricional del mismo. En la presente investigación se evidenció que, en trópico alto de Nariño, 60 días es la edad más acertada de cosecha, lo que coincide con la anterior recomendación. En ese sentido un estudio demostró, que a mayor edad de la planta menor es el contenido de proteína cruda y digestibilidad, y mayores los niveles de FDA y FDN (Navarro y Rodríguez, 1990), lo cual es similar a la tendencia encontrada en la presente investigación tanto para T. diversifolia como para S. nigra.

\section{Conclusiones}

El factor edad de corte influyó sobre la producción y capacidad de recuperación de las especies Tithonia diversifolia y Sambucus nigra, lo que indica un comportamiento sobresaliente a los 80 y 90 días de corte, respectivamente. En cuanto a la calidad nutricional del forraje, para esta investigación se obtuvieron mejores valores a los 60 días de corte para ambas especies.

En el factor altura de corte no se presentaron diferencias significativas en las variables evaluadas para cada especie. Las especies evaluadas presentaron un alto porcentaje de proteína con moderadas cantidades de fibra, alta digestibilidad y buenas fracciones de minerales macro, especialmente cuando se realizó su cosecha a edad de 60 días, convirtiéndose en una buena alternativa nutricional, que permitiría disminuir costos de producción, asociado a una disminución en la compra de suplementos alimenticios para el ganado. 
Dentro de esta investigación, la especie $T$. diversifolia se encontraba en el límite de su adaptación altitudinal; sin embargo, presentó un óptimo comportamiento bajo diseño silvopastoril de banco forrajero. Se recomienda seguir estudiando estas especies con potencial nutricional y ambiental para el trópico alto de Colombia.

\section{Agradecimiento}

Los autores agradecen a la Corporación Colombiana de Investigación Agropecuaria (AGROSAVIA) donde se desarrolló el proyecto "Mejoramiento de la oferta forrajera, optimización de sistemas de alimentación y aseguramiento de la calidad e inocuidad de leche en el trópico alto del departamento de Nariño", financiado por el Sistema General de Regalías (SGR). “

\section{Literatura citada}

Apráez, J.E., J.M. Delgado, y J.P. Narváez. 2012. Composición nutricional, degradación in vitro y potencial de producción de gas, de herbáceas, arbóreas y arbustivas encontradas en el trópico alto de Nariño. Livest. Res. Rural Dev. 24:3. http:// www.lrrd.org/lrrd24/3/apra24044.htm (consultado 22 dic. 2018).

Banakar, P., N. Anand, C. Shashank, and Y. Neeti. 2018. Physically effective fiber in ruminant nutrition: A review. J. Pharm. Phytochem. 7:303-308.

Barreto, C., y D. Chamorro. 2005. Evaluación nutricional de Sambucus nigra en tres frecuencias de corte asociado con Pennnisetum clandestinum y predicción de la respuesta animal de hatos lecheros de Cundinamarca aplicando el sistema CNCPS. Tesis Lic., Universidad de Ciencias Aplicadas y Ambientales, Bogotá D.C, COL.

Bernal, E.J. 2003. Pastos y forrajes tropicales: Producción y manejo. 4ª ed. Ángel Agro, Ideagro, Botogá, COL.

Blanco, M., y M. Sierra. 2005. Caracterización bromatológica y evaluación de diferentes niveles de inclusión de Morera (Morus alba L) y Sauco (Sambucus nigra L) en la alimentación de conejos en ceba. Tesis Lic., Universidad de la Salle, Bogotá, COL.

Bragachini, M., P. Cattani, M. Gallardo, y J. Peiretti. 2008. Forrajes conservados de alta calidad y aspectos relacionados al manejo nutricional. Manual técnico 6. 2d $\mathrm{d}^{\mathrm{a}}$ ed. Instituto Nacional de Tecnología Agropecuaria, Cordoba, ARG.

Calle, Z., y E. Murgueitio. 2014. El botón de oro: arbusto de gran utilidad para sistemas ganaderos de tierra caliente y de montaña. Rev. Carta Fedegan 108:51-62.

Cárdenas, C., C. Rocha, y R. Castaño. 2016. Efecto de la edad sobre las medidas morfométricas la composición bromatológica y el fraccionamiento de la proteína del sauco (Sambucus nigra). Agron. Costarricense 40:107-116. doi:10.15517/rac. v40i2.27390

Cárdenas, C., C. Rocha, y J. Mora. 2011. Productividad y preferencia de forraje de vacas lecheras pastoreando un sistema silvopastoril intensivo de la zona alto Andina de Roncesvalles, Tolima. Rev. Colom. Cienc. Anim. 4(1):29-35.

Cardona-Iglesias, J., L. Mahecha-Ledesma, y J. Angulo-Arizala. 2017. Efecto sobre la fermentación in vitro de mezclas de Tithonia diversifolia, Cenchrus clandestinum y grasas poliinsaturadas. Agron. Mesoam. 28:405-426. doi:10.15517/ ma.v28i2.25697

Carvajal, T., L. Lamela., y A. Cuesta. 2012. Evaluación de las arbóreas Sambucus nigra y Acacia decurrens como suplemento para vacas lecheras en la Sabana de Bogotá, Colombia. Pastos y Forrajes 35:417-430. 
Carvajal, T., y A. Cuesta. 2016. Conservación y composición nutricional del follaje de sauco (Sambucus nigra). Pastos y Forrajes. 39:125-132.

Cortés, K., y L. Ramos. 2018. Caracterización nutricional y antinutricional de algunas especies forrajeras promisorias para alimentación animal en el municipio de Pasto. Tesis Esp., Universidad Nacional Abierta y a Distancia, Bogotá, COL.

Corvalán, P., y J. Hernández. 2012. Medición de copas y raíces. En P. Corvalán, y J. Hernández, Apuntes de Dendrometría. Universidad de Chile, Santiago de Chile, CHI. p. 1-6.

Escobar-Pachajoa, L., C. Guatusmal-Gelpud, D. Meneses-Buitrago, J.L. Cardona-Iglesias, y E. Castro-Rincón. 2019. Evaluación de estratos arbóreos y arbustivos en un sistema silvopastoril en el trópico altoandino colombiano. Agron. Mesoam. 30:803-819. doi:10.15517/am.v30i3.35645

Ella, A., G.J. Blair, and W.W. Stür. 1991. Effect of age of forage tree legumes at the first cutting on subsequent production. Trop. Grassl. 25:275-280.

Fox, J., and S. Weisberg. 2011. The car package contains functions and data sets associated with the book an R companion to applied regression. $3^{\text {rd }}$ ed. R-Project. https://r-forge.r-project.org/projects/car/https://CRAN.R-project.org/package=car (accessed Jan. 10, 2019)

Gallego, L., L. Mahecha, y J. Angulo. 2015. Crecimiento y desarrollo de Tithonia diversifolia Hemsl. A Gray en condiciones de trópico alto. En: P. Pietri, editor, $3^{\circ}$ Congreso Nacional de Sistemas Silvopastoriles, VIII Congreso Internacional de Sistemas Agroforestales, Misiones, Argentina. Santa Cruz Ediciones, INTA, ARG. p. 53-57.

Gallego-Castro, L., L. Mahecha-Ledesma, y J. Angulo-Arizala. 2014. Potencial forrajero de Tithonia diversifolia Hemsl. A Gray en la producción de vacas lecheras. Agron. Mesoam. 25:393-403. doi:10.15517/am.v25i2.15454

Gallego-Castro, L., L. Mahecha-Ledesma, y J. Ángulo-Arizala. 2017. Calidad nutricional de Tithonia diversifolia Hemsl. A Gray bajo tres sistemas de siembra en el trópico alto. Agron. Mesoam. 28: 213-222. doi:10.15517/am.v28i1.21671

García, D., y N. Ortegón. 2014. Producción de biomasa y calidad nutricional de la asociación pasto kikuyo (Pennisetum clandestinum) y sauco (Sambucus nigra) en el trópico alto. Tesis Lic., Universidad de la Salle, Bogotá D.C., COL.

Grajales, B.M., M.M. Botero, y J.F. Ramírez. 2015. Características, manejo, usos y beneficios del saúco (Sambucus nigra L.) con énfasis en su implementación en sistemas silvopastoriles del Trópico Alto. Rev. Investig. Agrar. Ambient. 6(1):155168. doi: $10.22490 / 21456453.1271$

Jama, B., C.Palm, R.Buresh,A.Niang, C. Gachengo, G.Nziguheba, and B.Amadalo. 2000. Tithonia diversifolia as a green manure for soil fertility improvement in western Kenya A review. Agrofor. Syst. 49:201-221. doi:10.1023/A:1006339025728

Jaramillo, C. 2006. Evaluación del crecimiento de terneras Holstein con la suplementación de dos niveles de Saúco (S. nigra) en pastoreo con kikuyo (P. clandestinum). Rev. Invest. 6(2):189-197.

Jaramillo, Y., y J. Jiménez. 2000. Evaluación nutricional de tres especies de árboles forrajeros en la alimentación de vacas Holstein en el trópico alto de Nariño. Tesis Lic., Universidad de Nariño, Pasto, COL.

Lezcano, Y., M. Soca, F. Ojeda, E. Roque, D. Fontes, I. Montejo, H. Santana, J. Martínez, y N. Cubillas. 2012. Caracterización bromatológica de Tithonia diversifolia (Hemsl.) A. Gray en dos etapas de su ciclo fisiológico. Pastos y Forrajes 35:275282 .

Londoño, C., L. Mahecha, y J. Angulo. 2019. Desempeño agronómico y valor nutritivo para la alimentación de bovinos de Tithonia diversifolia (Hemsl.) A Gray. RECIA 11(1). doi:10.24188/recia.v0.n0.2019.693

López, J., y S. Santamaría. 2003. Evaluación de la producción de biomasa de tres especies forrajeras en un banco de proteína en el C.I. Obonuco, Municipio de Pasto. Tesis Lic., Universidad de Nariño, Pasto, COL. 
Lugo, M., F. Molina, I. Gonzáles, J. Gonzáles, y E. Sánchez. 2012. Efecto de la altura y frecuencia de corte sobre la producción de materia seca y proteína bruta de Tithonia diversifolia. Rev. Zootec. Trop. 30:317-325.

Mahecha, L., L. Gallego, y F. Peláez. 2002. Situación actual de la ganadería de carne en Colombia y alternativas para impulsar su competitividad y sostenibilidad. Rev. Colom. Cienc. Pecu. 15:213-225.

Mahecha, L., y M. Rosales. 2005. Valor nutricional del follaje de Botón de Oro (Tithonia diversifolia [Hemsl]. Gray) en la producción animal en el trópico. Livest. Res. Rural Dev. 17:9. https://www.lrrd.cipav.org.co/lrrd17/9/mahe17100.htm (consultado 20 dic. 2018).

Matta, S.L. 2005. Estrategias modernas para la conservación de forrajes en sistemas de producción bovina tropical. Corpoica. Cienc. Tecnol. Agropecu. 6(2):69-80.

Medina, M., D. García, L. Gonzales, y P. Moratinos. 2009. Variables morfo-estructurales y de calidad de la biomasa de Tithonia diversifolia en la etapa inicial de crecimiento. Zoot. Trop. 27(2):121-134.

Mendiburu, F. 2017. Agricolae. Statistical procedures for agricultural research. R package version 1.2-8. R-Project. https:// cran.r-project.org/web/packages/agricolae/index.html (accessed Sep. 10, 2018).

Millán, H., y F.W. Moreno. 2005. Evaluación de adaptación al establecimiento en arbóreas multipropósito para sistemas ganaderos sostenibles en la Sabana de Bogotá. Tesis Lic., Universidad Nacional de Colombia, Bogotá D.C., COL.

Mohan, B., and P.K. Ramachandran (ed.). 2011. Carbon sequestration potential of agroforestry: Opportunities and challenges. Springer, FL, USA. doi:10.1007/978-94-007-1630-8

Molina, A. 2016. Evaluación de especies arbustivas forrajeras con potencial para el diseño de estrategias de adaptación de los sistemas de producción ganadera al cambio climático, en ecosistemas de bosque húmedo tropical. Tesis Lic., Universidad de la Salle, Bogotá D.C., COL.

Morales, A., J. León, E. Cárdenas, G. Afanador, y J. Carulla. 2013. Calidad de la leche, digestibilidad in vitro de la materia seca y producción de vacas alimentadas con gramíneas solas o asociadas con Lotus uliginosus. Rev. Med. Vet. Zootec. 60:32-48.

Muhammad, I., A. Camero, J. Camargo, y H. Jair. 1999. Sistemas silvopastoriles en América Central: Experiencias de CATIE. CATIE, Turrialba, CRI. http://repositorio.bibliotecaorton.catie.ac.cr/bitstream/handle/11554/4026/Sistemas_ silvopastoriles_en_America.pdf?sequence=1\&isAllowed=y (consultado 13 feb. 2019).

Murgueitio, E., Z. Calle, F. Uribe, A. Calle, and B. Solorio. 2011. Native trees and shrubs for the productive rehabilitation of tropical cattle ranching lands. For. Ecol. Manag. 261:1654-1663. doi:10.1016/j.foreco.2010.09.027

NRC (National Research Council). 2001. The nutrient requirement of dairy cattle. $7^{\text {th }}$ ed. National Academy Press, WA, USA.

Navarro, F., and E. Rodríguez. 1990. Study of several bromatological aspects of Mirasol (Tithonia diversifolia Hemsl y Gray) as a potential alternative for animal feeding. Lic. Thesis. Universidad del Tolima, Ibague, COL.

Parada, J. 2006. Evaluación del potencial forrajero de botón de oro (Tithonia diversifolia (Hemsl.) A. Gray y Cayeno Hibiscus rosa-sinensis Linneo a diferentes densidades de siembra en el Municipio de Chinacota (Norte de Santander). Tesis MSc., Universidad Francisco de Paula Santander, Santander, COL.

Partey, S. 2011. Effect of pruning frequency and pruning height on the biomass production of Tithonia diversifolia (Hemsl) A. Gray. Agrof Syst 83:181. doi:10.1007/s10457-010-9367-y

R Development Core Team. 2008. R: A language and environment for statistical computing. R Foundation for Statistical Computing, Vienna, AUS. http://www.icesi.edu.co/CRAN/bin/windows/base/old/3.5.1/ (accessed Jan. 10, 2019).

Rawson, H., y H. Gómez. 2001. Factores ambientales: efectos de la temperatura. En: FAO, editor, Trigo regado: manejo de cultivo. FAO, Roma, ITA. p. 27-31. 
Rivera, J., C. Cuartas, J. Naranjo, O. Tafur, E. Hurtado, F. Arenas, J. Chará, y E. Murgueitio. 2015. Efecto de la oferta y el consumo de Tithonia diversifolia en un sistema silvopastoril intensivo (SSPi), en la calidad y productividad de leche bovina en el piedemonte Amazónico colombiano. Livest. Res. Rural Dev. 27:10. http://www.lrrd.org/lrrd27/10/rive27189. html (consultado 20 dic. 2018).

Roncallo, F., M. Sierra, y E. Castro. 2012. Rendimiento de forraje de gramíneas de corte y efecto sobre calidad composicional y producción de leche en el Caribe seco. Corpoica. Cienc. Tecnol. Agropecu. 13:71-78. doi:10.21930/rcta.vol13_num1_ art:242

Ruiz, T., G. Febles, y H. Díaz. 2012. Distancia de plantación, frecuencia y altura de corte en la producción de biomasa de Tithonia diversifolia colecta 10 durante el año. Rev. Cub. Cien. Agríc. 46:423-426.

Sanabria, E., e I. Ávila. 2015. Producción de follaje de la especie botón de oro (Tithonia diversifolia) utilizando 5 técnicas de siembra con fines de alimentación animal. Tesis Lic., Universidad Nacional Abierta y a Distancia, Bogotá D.C., COL.

Sánchez, L., G. Amado, P. Criollo, T. Carvajal, J. Roa, A. Cuesta, A. Conde, A. Umaña, L. Bernal, y L. Barreto. 2010. El Saúco (Sambucus nigra L) como alternativa silvopastoril en el manejo sostenible de praderas en el trópico alto Colombiano. Corpoica, COL.

Santacoloma, L.E. 2011. Las dietas en las emisiones de metano durante el proceso de rumia en sistemas de producción bovina. RIIA 2(1):55-64. doi:10.22490/21456453.913

Stür, W., H. Shelton, and R. Gutteridge. 1994. Defoliation management of forage tree legumes. In: R.C. Gutteridgeand, and H.M. Shelton, editors, Forage tree legumes in tropical agriculture. CAB International, Wallingford, GBR. p. $158-167$.

Téllez, A., y R. Mendoza. 2014. Comportamiento productivo de Tithonia diversifolia en bancos forrajeros, bajo condiciones de suelos de piedemonte llanero. Tesis Lic., Universidad de la Salle, Bogotá D.C., COL.

Van-Soest, P. 1994. Nutritional ecology of the ruminant. $2^{\text {nd }}$ ed. Cornell University Press, Ithaca, and London, GBR.

Verdecia, D., J. Ramírez, I. Leonard, Y. Álvarez, Y. Bazán, R. Bodas, S. Andrés, J. Álvarez, F. Giráldez, y S. López. 2011. Calidad de la Tithonia diversifolia en una zona del Valle del Cauto. REDVET 12(5). http://www.veterinaria.org/revistas/ redvet/n050511/051113.pdf (consultado 22 dic. 2018).

Zapata, Á., y J.E Vargas. 2014. Botón de oro: Manual para su establecimiento y manejo en sistemas ganaderos. Universidad de Caldas, Manizales, Caldas, COL. 